\title{
Electrochemical impedance spectroscopy and fluorescence lifetime imaging of lipid mixtures self-assembled on mercury $\dagger$
}

\author{
Lucia Becucci, ${ }^{* a}$ Stefano Martinuzzi, ${ }^{a}$ Emanuela Monetti, ${ }^{a}$ Raffaella Mercatelli, ${ }^{b}$ Franco Quercioli, ${ }^{b}$ \\ Dario Battistel $^{c}$ and Rolando Guidelli ${ }^{a}$
}

\author{
Received 12th November 2009, Accepted 11th March 2010 \\ First published as an Advance Article on the web 7th May 2010 \\ DOI: $10.1039 / b 923895 f$
}

\begin{abstract}
Monolayers and bilayers of lipid mixtures self-assembled on mercury form spontaneously gel-phase (solid ordered, $s_{\mathrm{o}}$ ) and liquid-ordered $\left(l_{\mathrm{o}}\right)$ microdomains, thanks to the fluidity imparted to these films by the liquid metal support. The differential capacity of the hydrocarbon tail region of monolayers of mixtures of two lipid components of high and low transition temperature $T_{\mathrm{m}}$, increases during the transition from the liquid disordered $\left(l_{\mathrm{d}}\right)$ phase to the coexistence of $l_{\mathrm{d}}$ and $s_{\mathrm{o}}$ phases. Addition of cholesterol to this binary mixture causes a decrease in differential capacity. This behavior is explained by regarding the capacity as a measure of the total perimeter of the $s_{\mathrm{o}}$ microdomains, due to the mismatch between these microdomains and the $l_{\mathrm{d}}$ phase. Cholesterol removes this mismatch by converting the anisotropic $s_{\mathrm{o}}$ microdomains into isotropic $l_{\mathrm{o}}$ microdomains (rafts). This allows differential capacity measurements by electrochemical impedance spectroscopy to follow phase transitions in lipid mixtures. The coexistance of $l_{\mathrm{d}}, l_{\mathrm{o}}$ and $s_{\mathrm{o}}$ phases is confirmed by images of a distal lipid monolayer self-assembled on top of a thiolipid monolayer tethered to a mercury microcap, by using two-photon fluorescence lifetime imaging microscopy (2P-FLIM).
\end{abstract}

\section{Introduction}

During the past decade membrane models studies have shown that phase separation can occur in binary lipid mixtures consisting of lipids with melting temperatures $T_{\mathrm{m}}$ respectively higher and lower than room temperature. Typically, gel phase microdomains, enriched in the high- $T_{\mathrm{m}}$ component, are surrounded by a liquid-crystalline phase enriched in the low- $T_{\mathrm{m}}$ component. ${ }^{1,2}$ The gel phase is anisotropic, tightly packed and has limited lateral mobility and axial rotation of the acyl chains. Conversely, the liquid-crystalline phase is isotropic, loosely packed and has a high degree of lateral mobility and axial rotation. Addition of cholesterol (Chol) to these binary mixtures has a disordering effect on the gel phase and an ordering effect on the liquidcrystalline phase. More precisely, in the liquid-crystalline phase cholesterol decreases the axial rotation of acyl chains and, at concentrations above $30 \mathrm{~mol} \%$, it also decreases lateral diffusion. Conversely, Chol increases lateral diffusion and axial rotation in the gel phase microdomains, causing them to become isotropic. ${ }^{3}$

${ }^{a}$ Department of Chemistry, Florence University, Via della Lastruccia 3, 50019 Sesto Fiorentino, Firenze, Italy. E-mail: lucia.becucci@unifi.it; Fax: (+39) 055-457-3385; Tel: (+39) 055-457-3095

${ }^{b} I S C$, Istituto dei Sistemi Complessi del CNR, CNR, Via Madonna del Piano, 10, 50019 Sesto Fiorentino, Firenze, Italy

${ }^{c}$ Department of Physical Chemistry, Venice University, Calle Larga S. Marta 2137, 30123 Venezia, Italy

$\dagger$ Electronic supplementary information (ESI) available: Three different types of plot commonly employed to display experimental impedance spectra are examined and compared. Exemplificative electrochemical impedance spectra of mercury-supported DOPC/PSM monolayers and of DPTL/phospholipid bilayers are reported. The rationale behind the use of an equivalent circuit consisting of a series of RC meshes for the fitting of impedance spectra of supported self-assembled layers is described. See DOI: 10.1039/b923895f
This is revealed by a morphological investigation of these lipid mixtures by two-photon excitation fluorescence microscopy. ${ }^{4}$ Thus, the gel phase microdomains have irregular shapes due to their anisotropic structure. Conversely, the microdomains in the presence of Chol concentrations higher than about $6 \mathrm{~mol} \%$, have a roundish shape, because they are isotropic like the surrounding liquid-crystalline phase. In this case, the line tension (the energy per unit length of the edge) associated with the edge of the two demixing phases tends to a minimum by optimizing the area-toperimeter ratio. In other words, Chol causes the microdomains to pass from the gel phase to the so-called "liquid ordered" $\left(l_{\mathrm{o}}\right)$ phase. This phase is regarded as "liquid" thanks to its sufficient lateral diffusion, and "ordered" because it is more tightly packed than the liquid-crystalline phase, due to the still modest axial rotation of its acyl chains. ${ }^{3}$ In contraposition with the $l_{\mathrm{o}}$ phase, the liquid-crystalline phase is often referred to as the "liquid disordered" $\left(l_{\mathrm{d}}\right)$ phase. Similarly, the gel phase is referred to as the solid ordered $\left(s_{\mathrm{o}}\right)$ phase.

Detergent-resistant biomembrane fractions, enriched in sphingolipids and Chol, have frequently been isolated using cold non-ionic detergents. ${ }^{1,2}$ Nowadays, it is generally accepted that these fractions are present in cell membranes as microdomains, called "lipid rafts". Lipid rafts are proposed to act as platforms for the preferential sorting of proteins ${ }^{5}$ that interact with each other to carry out cellular function, such as signal transduction. Lipid rafts are proposed to exist in a state similar to the $l_{\mathrm{o}}$ phase, surrounded by a $l_{\mathrm{d}}$ lipid matrix. Thus, sphingolipids have a high $T_{\mathrm{m}}$, while biological phospholipids have a low $T_{\mathrm{m}}$. Moreover, Chol accumulates preferentially in the $l_{\mathrm{o}}$ phase of membrane models.

Due to the important role ascribed to lipid rafts in cell signaling and molecular trafficking, raftlike $l_{\mathrm{o}}$ microdomains 
have been extensively investigated in different membrane models. Large and small unilamellar vesicles or multilamellar vesicles with different lipid mixtures have been investigated by twophoton fluorescence microscopy, ${ }^{4,6-9}$ fluorescence quenching, ${ }^{10}$ anisotropy imaging microscopy ${ }^{11}$ and small-angle neutron scattering. ${ }^{12}$ Free standing ${ }^{13}$ and glass supported planar lipid bilayers ${ }^{14}$ and living cells ${ }^{15}$ have also been examined by fluorescence microscopy. $L_{\mathrm{o}}$ microdomains of submicromolar dimensions formed on planar monolayers and bilayers supported by mica have been examined by AFM. ${ }^{16-18}$ Three-dimensional AFM images of phase-separated lipid bilayers supported by mica, with different microdomain height, were also reported. ${ }^{19}$ Electrochemical investigations on the coexistence of different lipid phases in membrane models are practically absent. Figaszewski et al. investigated binary mixtures of different lipids on free standing planar lipid bilayers by electrochemical impedance spectroscopy (EIS). However, their interest was mainly focused on the stoichiometry of specific complexes between lipids $(1: 3$ molecular ratio for ergosterol/egg PC; ${ }^{20} 1: 1$ for Chol/egg PC; $1: 10$ for $\alpha$-tocopherol/egg $\mathrm{PC}^{22}$ ) rather than on phase coexistence. Clearly, the area occupied by a single molecular complex, called "domain" by Figaszewski, cannot be identified with that of a microdomain.

Metal-supported membrane models having a lateral mobility comparable with that of biomembranes are obtained by selfassembling lipid monolayers or bilayers on mercury, thanks to its liquid nature. Lipid monolayers are readily self-assembled on the hydrophobic mercury surface, with the hydrocarbon tails directed toward the metal and the polar heads directed toward the aqueous solution. ${ }^{23-25}$ Lipid bilayers can be formed by tethering a thiolipid monolayer to the mercury surface. A convenient thiolipid, called DPTL, consists of a hydrophilic tetraethyleneoxy "spacer" terminated at one end with a lipoic acid residue, for anchoring to the mercury surface, and covalently linked at the other end to two phytanyl chains simulating the hydrocarbon tails of a lipid. By self-assembling a lipid monolayer on top of the thiolipid monolayer, a lipid bilayer is obtained, interposed between the hydrophilic spacer and the aqueous solution. ${ }^{26-30}$ Indirect evidence for the lateral mobility of this mercury-supported "tethered bilayer lipid membrane" (tBLM) is provided by its capability of incorporating in a functionally active state bulky channel proteins, such as OmpF porin ${ }^{27}$ and the HERG potassium channel. ${ }^{28}$

This work reports an EIS investigation of $\mathrm{Hg}$-supported monolayers consisting of binary mixtures of a low- $T_{\mathrm{m}}$ and a high$T_{\mathrm{m}}$ lipid, both in the absence and in the presence of Chol. The choice of lipid monolayers for EIS measurements is dictated by the large percentage changes of electrochemical impedance with varying the composition of a lipid monolayer, when it is directly self-assembled on a mercury electrode. Conversely, if the lipid monolayer is self-assembled on top of a DPTL monolayer anchored to mercury, much of the resistance and capacity of the resulting mixed bilayer is due to the DPTL monolayer. This work also reports images of $l_{\mathrm{o}}$ and gel phase microdomains in the distal lipid monolayer of $\mathrm{Hg}$-supported tBLMs, as obtained by two photon fluorescence lifetime imaging microscopy (2P-FLIM). For imaging measurements, mercury-supported tBLMs are to be preferred to mercury-supported lipid monolayers. A mercury support quenches fluorescence only slightly, thanks to its perfectly smooth surface. ${ }^{31}$ However, fluorescence quenching becomes completely negligible if the fluorophore is located in the polar head region of the distal lipid monolayer of a mercury-supported tBLM, at a distance of about $8 \mathrm{~nm}$ from the mercury surface.

\section{Experimental}

\section{Chemicals}

The water used was obtained from water produced by an inverted osmosis unit, upon distilling it once and then distilling the water so obtained from alkaline permanganate. Merck (Milano, Italy) suprapur ${ }^{\circledR} \mathrm{KCl}$ was baked at $500{ }^{\circ} \mathrm{C}$ before use to remove any organic impurities. Dipalmitoylphosphatidylcholine (DPPC; melting temperature $T_{\mathrm{m}}=41{ }^{\circ} \mathrm{C}$ ), diphytanoylphosphatidylcholine (DPhyPC; $T_{\mathrm{m}}<-120{ }^{\circ} \mathrm{C}$ ) and palmitoylsphingomyelin (PSM; $T_{\mathrm{m}}=41{ }^{\circ} \mathrm{C}$ ) were purchased in chloroform solution from Avanti Polar Lipids (Birmingham, AL). Dioleoylphosphatidylcholine (DOPC; $T_{\mathrm{m}}=-20{ }^{\circ} \mathrm{C}$ ) was obtained from Lipid Products (South Nutfield, Surrey, U.K.). Cholesterol (Chol) was purchased from Sigma (Milano, Italy) and used without further purification. DPTL (2,3,di- $O$-phytanyl$s n$-glycerol-1-tetraethylene-glycol-D,L- $\alpha$ lipoic acid ester) was provided by Prof. Adrian Schwan (Department of Chemistry, University of Guelph, Canada). Solutions of $0.2 \mathrm{mg} \mathrm{mL}^{-1}$ DPTL in ethanol were prepared from a $2 \mathrm{mg} \mathrm{mL}^{-1}$ solution of DPTL in ethanol. Stock solutions of this thiolipid were stored at $-18^{\circ} \mathrm{C}$.

\section{Preparation of the biomimetic membranes}

All measurements were carried out in aqueous $0.1 \mathrm{M} \mathrm{KCl}$. Use was made of a home-made hanging mercury drop electrode (HMDE), described elsewhere. ${ }^{32}$ A home-made glass capillary with a finely tapered tip, about $1 \mathrm{~mm}$ in outer diameter, was employed. Capillary and mercury reservoir were thermostated at $25 \pm 0.1^{\circ} \mathrm{C}$ in a water-jacketed box to avoid any changes in drop area due to a change in temperature. Lipid monolayers were selfassembled on mercury as described earlier. ${ }^{23-25}$ Briefly, the appropriate lipid mixture in pentane was spread on the surface of the working $0.1 \mathrm{M} \mathrm{KCl}$ aqueous solution and the pentane was allowed to evaporate. The HMDE was then immersed slowly in the solution across the lipid film, under the control of an oleodynamic system. This procedure gives rise to a self-assembled lipid monolayer, with the hydrocarbon tails directed towards the hydrophobic mercury surface. The lipid monolayer was stabilized by scanning the applied potential $E$ repeatedly between -0.2 and $-0.8 \mathrm{~V}$, while continuously monitoring the quadrature component of the electrode admittance, $Y^{\prime \prime}$, by a.c. voltammetry at a frequency of $75 \mathrm{~Hz}$; the scanning was stopped when a stable $Y^{\prime \prime}$ versus potential curve was attained. Finally, the electrochemical impedance spectrum was measured at $-0.550 \mathrm{~V}$ over the frequency range from 0.1 to $10^{5} \mathrm{~Hz}$. These measurements were repeated several times in the same cell on newly formed drops by following the same procedure.

Imaging measurements by 2P-FLIM were carried out on a tethered bilayer lipid micromembrane $(\mathrm{tBL} \mu \mathrm{M})$ described in Ref. 33. Briefly, the tBL $\mu \mathrm{M}$ was obtained from a platinum wire, about $20 \mu \mathrm{m}$ in diameter, which was sealed in a glass capillary, $1 \mathrm{~mm}$ in outer diameter, and terminated with a platinum microdisk. Mercury was electrodeposited on the microdisk from 
a deaerated $\mathrm{pH} 1$ solution of $10 \mathrm{mM} \mathrm{Hg} 2\left(\mathrm{NO}_{3}\right)_{2}$ to form a mercury spherical cap. The electrodeposition was monitored by recording the resulting faradaic charge against time and was interrupted when the charge attained the desired value. The Hgcoated Pt microdisk was immersed in a DPTL solution in ethanol for about $20 \mathrm{~min}$, to anchor a DPTL monolayer to the $\mathrm{Hg}$ surface, and then washed with ethanol to remove the DPTL in excess. To form a lipid monolayer on top of the DPTL monolayer, a few drops of a solution of the appropriate lipid mixture in chloroform were then spilled on the tip of the microelectrode, allowing the solvent to evaporate. The resulting tBL $\mu \mathrm{M}$ was stabilized in aqueous $0.1 \mathrm{M} \mathrm{KCl}$ by continuous cyclic voltammetric scans between -0.2 and $-1.2 \mathrm{~V}$.

\section{Instrumentation}

Electrochemical impedance spectroscopy measurements were carried out with an Autolab instrument PGSTAT12 (Echo Chemie, Utrecht, The Netherlands) supplied with FRA2 module for impedance measurements, SCAN-GEN scan generator and GPES 4.9007 software. Potentials were measured vs. a $\mathrm{Ag} \mid \mathrm{AgCl}$ electrode immersed in the $0.1 \mathrm{M} \mathrm{KCl}$ working solution and are referred to this electrode.

2P-FLIM images were recorded with a multiphoton microscope (MM) equipped with a time correlated single photon counting (TCSPC) system. The MM consisted of a Nikon PCM 2000 confocal laser scanning microscope (CLSM), with a Nikon Eclipse TE 2000-U inverted optical microscope (Tokyo, Japan). The Confocal Laser scanning unit was modified to allow the use of an Ultrafast Laser Source, by substituting the original longpass dichroic beamsplitter with a short-pass one (650DCSPXR, Chroma Inc., Brattelboro, USA), suitable for multiphoton operation. The laser source was a mode locked Ti:Sapphire oscillator (Mira 900 F, Coherent Inc., Santa Clara, CA) pumped by a $5 \mathrm{~W}$ frequency-doubled Nd:YVO4 Laser at $532 \mathrm{~nm}$ (Verdi V5, Coherent Inc., Santa Clara, CA). The Ti:Sapphire wavelength tunable range was $700-980 \mathrm{~nm}$, with X-Wave broadband optics, and its typical pulse duration was $130 \mathrm{fs}$, with a repetition rate of $76 \mathrm{MHz}$. The whole system was placed on an antivibration optical table and the laser source was directly coupled to the confocal scanning unit to avoid pulse broadening. The MM had two output channels, one of which was used for FLIM imaging. The fluorescence emission, once passed through a Schott BG-39 filter (Cambridge, UK) to completely cut down the Laser near infrared excitation, was sent to the TCSPC system (Becker \& Hickl GmbH, Edinburgh, UK). This consisted of a PMH-100, a fast photon counting photomultiplier that yielded a system response of 150 to 220 ps FWHM and could measure lifetimes down to 100-200 ps, and of a SPC-830, a high-resolution TCSPC imaging module on single PC board. A PHD-400-N photodiode module provided the synchronisation signal from the laser, as required for the time measurement of the individual photon detection events. For each pixel of the image, the arrival times of the individual fluorescence photons were measured at each excitation laser pulse and, ultimately, a decay curve histogram was built up from these individual time measurements. Data analysis by the SPC Image software (Becker \& Hickl, Edinburgh, UK) delivered the fluorescence decay times to each individual pixel of the image.
For this 2P-FLIM measurement, a special experimental set-up was devised. The microelectrode was mounted on a three-axis mechanical support with differential micrometric screws and placed upon the microscope stage. A Nikon CFI Fluor $60 \times \mathrm{W}$ (Tokyo, Japan), with a 1.00 numerical aperture (NA) and a working distance (WD) of $2.0 \mathrm{~mm}$, was mounted on the inverted microscope objective revolver. This was a water dipping objective, which was designed to work without a cover glass and could be immersed directly in an aqueous solution. A water drop was placed on the objective and the microelectrode was lowered until its tip, with the mercury-supported micromembrane, was immersed in the water drop. The final focusing adjustment was made with the microscope micrometric screw using a safe illumination around $550 \mathrm{~nm}$, which was well outside the fluorophore absorption band. The ultrafast laser source was tuned at a $2 \mathrm{P}$ excitation wavelength of $760 \mathrm{~nm}$. The acquisition time for each image was $180 \mathrm{~s}$ and the pixel dwell time $30 \mu \mathrm{s}$. This time duration was an appropriate compromise between the accumulation of a sufficient number of photons for lifetime statistics and an illumination short enough to avoid fluorophore photobleaching.

\section{Results}

\section{EIS measurements}

The inset of Fig. 1 shows the electrochemical impedance spectra of $\mathrm{Hg}$-supported monolayers of pure DOPC and of a DOPC/ $\operatorname{PSM}(50: 50)$ mixture, at $-0.550 \mathrm{~V}$ in aqueous $0.1 \mathrm{M} \mathrm{KCl}$. These spectra are displayed on a $\omega Z^{\prime}$ versus $\omega Z^{\prime \prime}$ plot, where $\omega$ is the angular frequency and $Z^{\prime}, Z^{\prime \prime}$ are the in-phase and quadrature components of the electrochemical impedance.

To interpret impedance spectra, it is necessary to compare them with the electrical response of an equivalent circuit

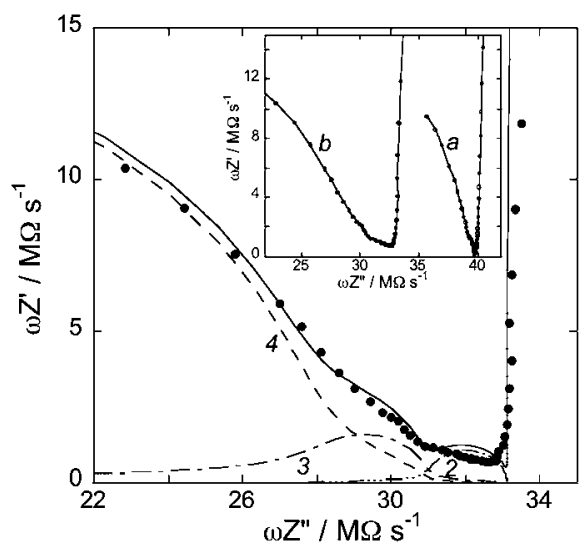

Fig. $1 \omega Z^{\prime}$ vs. $\omega Z^{\prime \prime}$ plot for a Hg-supported monolayer of the DOPC/ $\operatorname{PSM}(50: 50)$ mixture in aqueous $0.1 \mathrm{M} \mathrm{KCl}$ at $-0.550 \mathrm{~V}$. Solid circles are experimental points and the solid curve is the best fit to these points obtained with a series of four RC meshes. Curves 2 (- - - ) 3 (-.-) and 4 (---) are single $\omega Z^{\prime}$ contributions to this fit ascribable to two sections of the polar head region and to the hydrocarbon tail region, respectively. The capacities and resistances of the RC meshes 2, 3 and 4 are: $C_{2}=33 \mu \mathrm{F} \mathrm{cm}^{-2}, R_{2}=0.28 \mathrm{k} \Omega \mathrm{cm}^{2}, C_{3}=20 \mu \mathrm{F} \mathrm{cm}{ }^{-2}, R_{3}=18 \mathrm{k} \Omega \mathrm{cm}^{2}$, $C_{4}=2.8 \mu \mathrm{F} \mathrm{cm}^{-2}, R_{4}=3.5 \mathrm{M} \Omega \mathrm{cm}^{2}$. In the inset, curve $a$ is the $\omega Z^{\prime} v s$. $\omega Z^{\prime \prime}$ plot for a pure DOPC monolayer, while curve $b$ is the same plot as in the main part of the figure. 
simulating the system under investigation. In this respect, a metal-supported self-assembled mono- or multilayer can be conveniently regarded as consisting of a series of slabs with different dielectric properties. In an equivalent circuit, a dielectric slab can be represented as a resistance and a capacity in parallel, namely an RC mesh. Therefore, an impedance spectrum can be fitted by an equivalent circuit consisting of a series of RC meshes. In a $\omega Z^{\prime}$ versus $\omega Z^{\prime \prime}$ plot, which will be briefly referred to as an $\mathrm{M}$ plot (where $\mathrm{M}$ stands for modulus), a single RC mesh yields a semicircle of diameter $1 / C$; the angular frequency at the maximum of the semicircle equals the reciprocal of the time constant, RC, of the mesh. If the time constants of the different dielectric slabs are too close, the corresponding semicircles tend to overlap partly, giving rise to curves resembling depressed semicircular arcs on the $\mathrm{M}$ plot. Since the frequency increases along the positive direction of the $\omega Z^{\prime \prime}$ axis, the partially overlapping semicircles are aligned in the order of decreasing time constant, when proceeding in this direction.

Both $\mathrm{M}$ plots in the inset of Fig. 1 show an almost vertical section that is just the initial portion of a very large semicircle characterized by a very small capacity $C_{1}=36 \mathrm{nF} \mathrm{cm}^{-2}$ and a small resistance $R_{1}=4.2 \Omega \mathrm{cm}^{2}$, ascribable to the aqueous solution adjacent to the lipid monolayer. In addition to the above almost vertical section, the $\mathrm{M}$ plot of the DOPC monolayer (curve $a$ ) shows a portion of a semicircle and a small hump at its foot, which is due to a smaller semicircle almost completely overlapping with, and obliterated by, the larger one. The larger semicircle, of lower capacity, must be ascribed to the hydrocarbon tail region of the lipid monolayer, while the smaller one, of much higher capacity, is to be ascribed to the polar head region. Fitting this $\mathrm{M}$ plot to a series of three $\mathrm{RC}$ meshes yields the values $R_{2}=3.6 \mathrm{k} \Omega \mathrm{cm}^{2}, C_{2}=51 \mu \mathrm{F} \mathrm{cm}{ }^{-2}$ for the polar head region, and $R_{3}=3.3 \mathrm{M} \Omega \mathrm{cm}^{2}, C_{3}=1.9 \mu \mathrm{F} \mathrm{cm} \mathrm{cm}^{-2}$ for the hydrocarbon tail region; the third mesh is that, $\mathrm{R}_{1} \mathrm{C}_{1}$, due to the aqueous solution. In particular, the $C_{3}$ value of $1.9 \mu \mathrm{F} \mathrm{cm} \mathrm{cm}^{-2}$ is about twice that, $0.9-1 \mu \mathrm{F} \mathrm{cm} \mathrm{cm}^{-2}$, of solvent-free bilayer lipid membranes ${ }^{34}$ and in good agreement with the value of

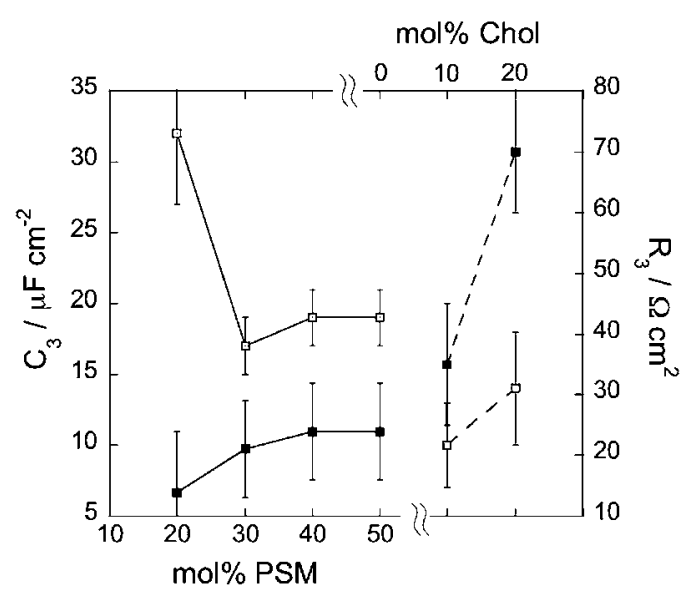

Fig. 2 A plot of the capacity $C_{3}(\square)$ and of the resistance $R_{3}(\boldsymbol{\square})$ for a $\mathrm{Hg}$ supported DOPC/PSM monolayer in aqueous $0.1 \mathrm{M} \mathrm{KCl}$ at $-0.550 \mathrm{~V}$, as a function of $\mathrm{mol} \% \mathrm{PSM}$. The effect of Chol addition to the DOPC/PSM $(50: 50)$ binary mixture is shown on the right side of the figure.

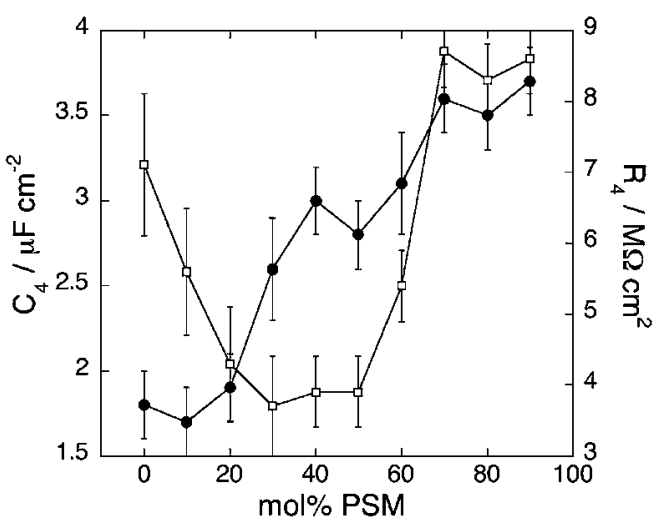

Fig. 3 A plot of the capacity $C_{4}(\bullet)$ and of the resistance $R_{4}(\square)$ for a $\mathrm{Hg}$-supported DOPC/PSM monolayer in aqueous $0.1 \mathrm{M} \mathrm{KCl}$ at $-0.550 \mathrm{~V}$, as a function of $\mathrm{mol} \% \mathrm{PSM}$.

$1.85 \mu \mathrm{F} \mathrm{cm}^{-2}$, reported for a mercury-supported DOPC monolayer self-assembled on mercury ${ }^{35}$ (for a review, see Ref. 36).

The $\mathrm{M}$ plot of the lipid mixture monolayer (curve $b$ ) differs from that of the pure DOPC monolayer by the presence of a flat region preceding a depressed semicircular arc. The minimum number of RC meshes in series simulating the $\mathrm{M}$ plot, with fitting errors less than $10 \%$ in the estimate of all $R$ and $C$ values, was found to be four. The rationale behind this fitting procedure is described in the ESI†. In Fig. 1, the calculated contribution to $\omega Z^{\prime}$ from each single $\mathrm{RC}$ mesh is plotted against the overall $\omega Z^{\prime \prime}$ quantity, with the exclusion of the omnipresent contribution from the aqueous solution. This serves to show how each semicircle is affected by partial overlapping with the neighboring ones. Each contribution is represented by a semicircle that the more distorted it is, the greater the overlap. Proceeding in the negative direction of the $\omega Z^{\prime \prime}$ axis, the first semicircle that one encounters after that due to the aqueous solution has a capacity of $C_{2}=35 \pm 10 \mu \mathrm{F} \mathrm{cm}{ }^{-2}$ and a resistance $R_{2}=0.25 \pm 0.05 \mathrm{k} \Omega$ $\mathrm{cm}^{2}$, which do not vary with an increase in the PSM mole fraction from 20 to $50 \mathrm{~mol} \%$. The immediately following semicircle has

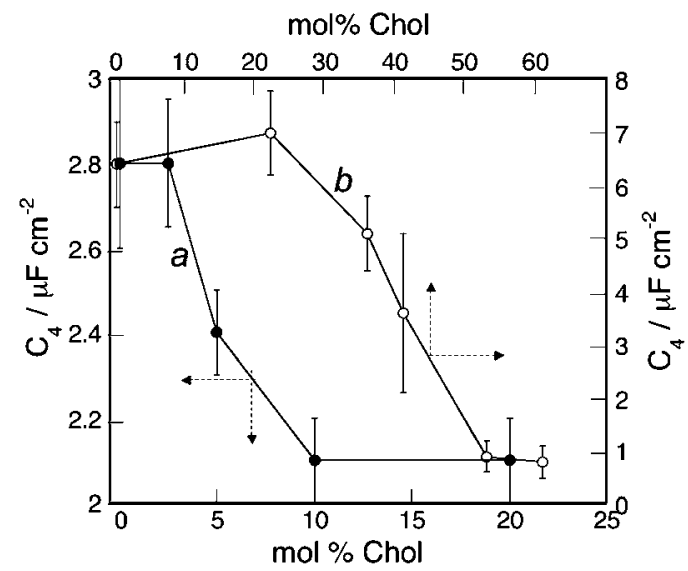

Fig. 4 A plot of the capacity $C_{4}$ : (a) for a $\mathrm{Hg}$-supported DOPC/PSM $(50: 50)$ monolayer in aqueous $0.1 \mathrm{M} \mathrm{KCl}$ at $-0.550 \mathrm{~V}$ as a function of the mole fraction of Chol added to it $(\bullet)$; (b) for a $\mathrm{Hg}$-supported PSM/ Chol monolayer in aqueous $0.1 \mathrm{M} \mathrm{KCl}$ at $-0.550 \mathrm{~V}$ as a function of $\mathrm{mol} \%$ Chol (O). 


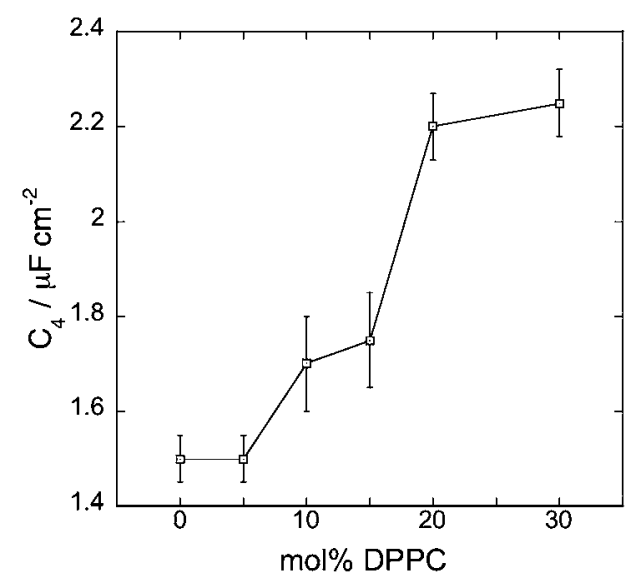

Fig. 5 A plot of the capacity $C_{4}(\square)$ for a Hg-supported DPhyPC/ DPPC monolayer in aqueous $0.1 \mathrm{M} \mathrm{KCl}$ at $-0.550 \mathrm{~V}$, as a function of $\mathrm{mol} \%$ DPPC.

a resistance, $R_{3}$, that increases and a capacity, $C_{3}$, that decreases with an increase in the PSM mole fraction, as shown in Fig. 2; a more pronounced increase in resistance and decrease in capacity is observed by adding Chol to the DOPC/PSM (50:50) mixture. The last semicircle is that of lowest capacity, $C_{4}$, apart from the large semicircle due to the aqueous solution. In analogy with the impedance spectrum for pure DOPC in the inset of Fig. 1, the corresponding $\mathrm{R}_{4} \mathrm{C}_{4}$ mesh is ascribed to the hydrocarbon tail region. The errors relative to the fitting of a single impedance spectrum to the four RC meshes in series lie below $10 \%$. However, the $R$ and $C$ values over several measurements on different mercury drops have larger fitting errors, which are represented by the error bars in Fig. 2 to 5. In fact, even though the surface area of the HMDE is expected to be much greater than that of the heterogeneities of the lipid mixture spread on the surface of the $0.1 \mathrm{M} \mathrm{KCl}$ aqueous solution, the composition of the monolayer self-assembling on the mercury drop may vary somewhat from one drop to another and from one spread lipid mixture to another.

Fig. 3 shows the $R_{4}$ and $C_{4}$ values of the DOPC/PSM binary mixture as a function of the mole fraction of PSM. As this mole fraction increases, the differential capacity $C_{4}$ undergoes an abrupt increase with a maximum slope at $\sim 25-30 \mathrm{~mol} \%$; then, with a further increase in the PSM mole fraction, $C_{4}$ shows a shallow minimum at $\sim 50 \mathrm{~mol} \%$ PSM, increases again with a maximum slope at $\sim 60 \mathrm{~mol} \% \mathrm{PSM}$, and then levels off. This plot ignores the $C_{4}$ value for pure PSM, which amounts to $\sim 6.5 \mu \mathrm{F} \mathrm{cm} \mathrm{cm}^{-2}$, and is therefore much higher than the values assumed over the 70 to $90 \mathrm{~mol} \%$ PSM range. While the first increase in $C_{4}$ is accompanied by a decrease in the corresponding resistance $R_{4}$, the second increase in $C_{4}$ is accompanied by an increase in $R_{4}$. Curve $a$ in Fig. 4 shows that the addition of $5 \mathrm{~mol} \%$ Chol to the DOPC/PSM (50:50) mixture causes an abrupt decrease in capacity. The resistance $R_{4}$ also shows a decrease (data not shown).

Analogous measurements of the capacity $C_{4}$ of the hydrocarbon tail region were carried out on the DPhyPC/DPPC binary mixture. The $C_{4}$ values are plotted in Fig. 5 against the DPPC mole fraction. Measurements were confined to mole fractions $\leq$ $30 \%$, because DPPC is scarcely soluble in pentane, which is the solvent used to spread the lipid mixture on the surface of the working electrolyte for the self-assembling procedure. In this case, an abrupt increase in $C_{4}$ is observed at $\sim 15-20 \mathrm{~mol} \%$ DPPC. EIS measurements were also carried out on the PSM/ Chol binary mixture. Curve (b) in Fig. 4 shows the $C_{4}$ value as a function of the Chol mole fraction. $C_{4}$ starts to decrease with respect to the high value for pure PSM at about $20 \mathrm{~mol} \% \mathrm{Chol}$, attaining a minimum final value at about $55 \mathrm{~mol} \% \mathrm{Chol}$.

\section{P-FLIM measurements}

An advantage of mercury over solid electrodes as a support of tBLMs is its very low fluorescence quenching efficiency. Thus, its perfectly smooth surface has a quenching efficiency lower than that of a polycrystalline Au surface of normal roughness, by two or three orders of magnitude. ${ }^{31}$ Even a phospholipid monolayer self-assembled on a HMDE and incorporating a fluorophore in its polar head region yields fluorescence images for a long period of time over the potential region of minimum capacity of the monolayer. ${ }^{37}$ Moreover, the fluorescent features of this mercurysupported monolayer, obtained by epi-fluorescence microscopy, are freely mobile, in contrast with those obtained on a gold support. Therefore, we found it interesting to obtain fluorescence lifetime images of a tethered bilayer lipid micromembrane $(\mathrm{tBL} \mu \mathrm{M})$ described in the materials and methods section. This consists of a mercury cap electrodeposited on a platinum microdisk and coated with a DPTL thiolipid monolayer, with a self-assembled monolayer of a lipid mixture on top of it. The diameter of the mercury cap is about equal to $20 \mu \mathrm{m}$, and is, therefore, comparable with that of giant unilamellar vesicles (GUVs). The liquid nature of mercury imparts to the thiolipid monolayer and to the overhanging lipid mixture fluidity and lateral mobility comparable with those of biomembranes, thus allowing the distal lipid mixture to form spontaneous microdomains in a very short time. Images of the distal lipid monolayer were obtained with a 2P-FLIM. To this end, the lipid mixture to be self-assembled on top of the thiolipid-coated mercury cap was labeled with $1 \mathrm{~mol} \%$ of the fluorophore laurdan.

The use of laurdan is particularly advantageous because it distributes uniformly in membranes, even in the presence of different coexisting lipid phases. ${ }^{4,6}$ Moreover, it is sensitive to the state of the lipid phase, as distinct from the majority of fluorescent probes. Thus, the water dipolar relaxation processes induced by laurdan photoexcitation decrease both the energy of the emitted photons and the fluorescence lifetime. This phenomenon is more pronounced in the polar head region of the $l_{\mathrm{d}}$ phase, where the water content is higher than in the $l_{\mathrm{o}}$ phase, and, even more so, in the gel phase. Consequently, laurdan emission is blue in the $l_{\mathrm{o}}$ phase and greenish in the $l_{\mathrm{d}}$ phase; moreover, its fluorescence lifetime is longer in the $l_{\mathrm{o}}$ phase than in the $l_{\mathrm{d}}$ phase. Since the two condensed aromatic rings of laurdan are located in the polar head region of the distal lipid monolayer, their distance from the mercury surface amounts to about $7.5-8 \mathrm{~nm} .^{38}$ Therefore, the non-radiative energy transfer from the photoexcited laurdan molecules to the perfectly smooth mercury surface is entirely negligible.

2P-FLIM images of two tBL $\mu \mathrm{M}$ distal monolayers, consisting of DOPC/PSM/Chol ternary mixtures of molar composition 
(70:15:15) and (47:47:6), labeled with $1 \mathrm{~mol} \%$ laurdan, are shown in Fig. 6 and 7. The images were recorded using $128 \times$ 128 pixels and 1024 time bins per pixel. For both lipid systems, monoexponential decays were observed. In the image of the DOPC/PSM/Chol $(70: 15: 15)$ mixture (see Fig. 6), the light blue roundish domains have lifetimes of $2750 \pm 50$ ps. Their roughly circular shape denotes the coexistence of two liquid phases, namely a $l_{\mathrm{o}}$ and a $l_{\mathrm{d}}$ phase. In fact, when an isotropic liquid domain is embedded in an isotropic liquid environment, it tends to assume a circular shape in order to minimize the line tension along its rim and to maximize its area-to-perimeter ratio. The light blue domains must be ascribed to the liquid phase of higher lifetime, and hence of higher order, namely the $l_{\mathrm{o}}$ phase typical of lipid rafts. As appears from the fluorescence lifetime distribution histogram, the orange areas have a fluorescence lifetime of about $2300 \mathrm{ps}$ and correspond to the $l_{\mathrm{d}}$ phase. Finally,
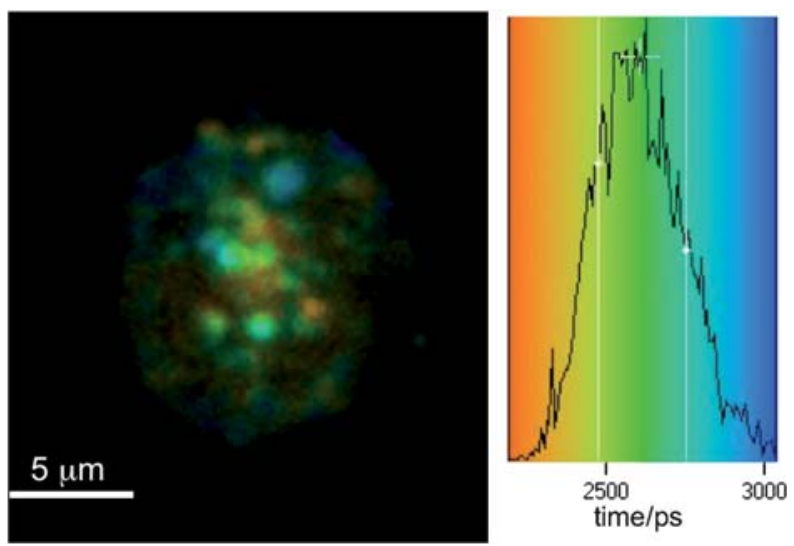

Fig. 6 A two-photon $(\lambda$ excitation $=760 \mathrm{~nm})$ fluorescence lifetime $21 \times$ $21 \mu \mathrm{m}$ image (left panel) and a fluorescence lifetime distribution histogram (right panel) of a DOPC/PSM/Chol $(70: 15: 15)$ mixture labeled with $1 \mathrm{~mol} \%$ laurdan, at room temperature. The mixture is the distal monolayer of a $\mathrm{tBL} \mu \mathrm{M}$. The color code in the FLIM image is that indicated in the histogram on the right.
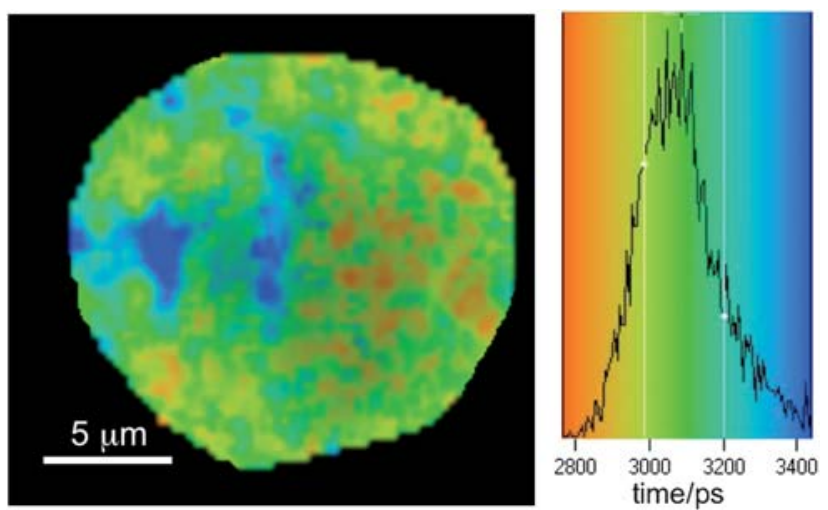

Fig. 7 A two-photon $(\lambda$ excitation $=760 \mathrm{~nm})$ fluorescence lifetime $21 \times$ $21 \mu \mathrm{m}$ image (left panel) and a fluorescence lifetime distribution histogram (right panel) of a distal monolayer of DOPC/PSM/Chol (47 : $47: 6$ ) mixture, labeled with $1 \mathrm{~mol} \%$ laurdan, at room temperature. The mixture is the distal monolayer of a tBL $\mu \mathrm{M}$. The color code in the FLIM image is that indicated in the histogram on the right. the greenish areas with intermediate fluorescence lifetimes are to be ascribed to the coexistence of the $l_{\mathrm{d}}$ phase and $l_{\mathrm{o}}$ microdomains of size below the resolution of the microscope (about $0.4 \mu \mathrm{m}$ radial). In this case the microdomain size is smaller than the image pixel size. The image of the DOPC/PSM/Chol (47:47:6) mixture in Fig. 7 shows a high degree of heterogeneity. The microdomains with an irregular percolative-like shape have lifetimes of $3450 \pm 50 \mathrm{ps}$ in the dark blue areas and of $3250 \pm 100 \mathrm{ps}$ in the light blue ones. Their shape agrees with that of FLIM images of similar lipid mixtures in GUVs; ${ }^{8}$ they must be ascribed to the gel phase. The small roundish orange microdomains immersed in the greenish surrounding matrix have lifetimes of $2830 \pm 50 \mathrm{ps}$ and should be attributed to the $l_{\mathrm{o}}$ phase. Therefore, the lipid mixture in Fig. 7 is characterized by the coexistence of $s_{\mathrm{o}}, l_{\mathrm{o}}$ and $l_{\mathrm{d}}$ phases.

\section{Discussion}

\section{Correlation between changes in differential capacity and phase transitions}

The RC meshes 2 and 3 in Fig. 1 can be tentatively ascribed to the polar head region of a mixture of lipids of different length, imagined to be subdivided into two ideal slabs parallel to the mercury surface, as shown schematically in Fig. 8. The slab closer to the electrode surface contains the polar heads of the shorterchain lipid (i.e. DOPC) as well as the distal portion of the hydrocarbon tails of the longer-chain lipid (i.e. PSM). This inner slab is expected to have a capacity lower and a resistance higher than that of the polar heads of pure DOPC. It can be ascribed to the $\mathrm{R}_{3} \mathrm{C}_{3}$ mesh. The addition of Chol thickens and stiffens this slab, thus causing a further decrease in its capacity $C_{3}$ and an increase in its resistance $R_{3}$, as actually shown in Fig. 2.

The outer slab contains the polar heads of the PSM molecules intercalated with water molecules. At PSM mole fractions of less than $20 \%$, this slab has a very low resistance $R_{2}$ and cannot be distinguished from the aqueous phase. Beyond this PSM mole fraction, the resistance of this outer slab becomes measurable,

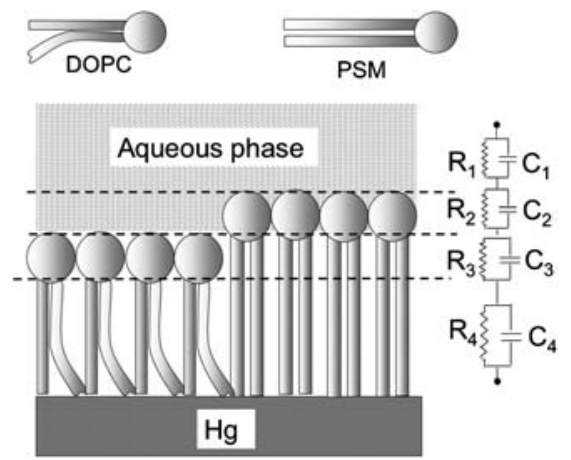

Fig. 8 A schematic picture of a raft-forming lipid mixture of DOPC and PSM molecules self-assembled on mercury, in which four slabs with different dielectric properties are envisaged. Slabs 1 to 4 comprise, in order, the aqueous solution adjacent to the lipid monolayer, the polar heads of PSM mixed with water molecules, the polar heads of DOPC mixed with the distal portion of the PSM hydrocarbon tails, and the hydrocarbon tails of both DOPC and PSM. The slabs ore simulated by four RC meshes in series. 
and it is coupled to a very high capacity $C_{2}$, due to the small thickness of the slab.

At any rate, the main interest is focused on the capacity $C_{4}$ and resistance $R_{4}$ of the slab of lower capacity, which can be safely ascribed to the lipid hydrocarbon tails and is determined with higher accuracy. The two rapid increases in $C_{4}$ in Fig. 3 lie at PSM mole fractions close to those marking the transitions from a $l_{\mathrm{d}}$ phase to a $s_{\mathrm{o}}+l_{\mathrm{d}}$ phase coexistence region, and from this to the $s_{\mathrm{o}}$ phase. Thus, the transition from the $l_{\mathrm{d}}$ phase to the $l_{\mathrm{d}}+s_{\mathrm{o}}$ two-phase region in the (palmitoyloleoylphosphatidylcholine = POPC)/PSM binary system at $23{ }^{\circ} \mathrm{C}$ was found to take place at $30 \mathrm{~mol}^{\%} \mathrm{PSM}$, on the basis of fluorescence anisotropy ${ }^{39}$ and time-resolved fluorescence resonance energy transfer (FRET) measurements. ${ }^{9,40}$ Moreover, the $l_{\mathrm{d}}+s_{\mathrm{o}}$ to $s_{\mathrm{o}}$ transition was reported to take place at about $67 \mathrm{~mol} \% \mathrm{PSM}^{40}$ Similarly, in the binary system consisting of the low- $T_{\mathrm{m}}$ component 1-dodecanoyl-2-(12-bromodecanoyl) phosphatidylcholine (12BrPC) and DPPC, the $l_{\mathrm{d}}$ to $l_{\mathrm{d}}+s_{\mathrm{o}}$ transition and the $l_{\mathrm{d}}+s_{\mathrm{o}}$ to $s_{\mathrm{o}}$ transition at $25{ }^{\circ} \mathrm{C}$ were estimated to take place at $20 \%$ and $65 \%$ DPPC, respectively, on the basis of infrared spectroscopy and fluorescence quenching measurements. ${ }^{41}$ Analogous considerations hold for the DPhyPC/DPPC system in Fig. 5. Here, the rapid increase in $C_{4}$ at $\sim 20 \mathrm{~mol} \%$ DPPC compares favorably with the transition from the $l_{\mathrm{d}}$ phase to a $s_{\mathrm{o}}+l_{\mathrm{d}}$ phase coexistence region, as reported at about the same mole fraction on the basis of fluorescence microscopy measurements. ${ }^{42}$ The lower mol fraction of the high- $T_{\mathrm{m}}$ component at which the $l_{\mathrm{d}}$ to $s_{\mathrm{o}}+l_{\mathrm{d}}$ transition takes place in the DPhyPC/DPPC system with respect to the DOPC/PSM system is to be ascribed to the very poor tightpacking ability of DPhyPC with respect to DOPC. Thus, DPhyPC has a multibranched structure, with four methyl groups that protrude from the main fatty acyl chain, and a $T_{\mathrm{m}}<-120^{\circ} \mathrm{C}$. This should result in it being much less miscible in tightly packed ordered domains than low- $T_{\mathrm{m}}$ lipids with less extreme poor packing properties, such as DOPC. ${ }^{43}$ Curve (b) in Fig. 4 shows the relatively slow change in the capacity $C_{4}$ from the value typical of pure PSM to that of Chol, which takes place between 25 and $50 \mathrm{~mol}_{\%} \mathrm{Chol}$.

The rapid decrease in $C_{4}$ after addition of Chol to the DOPCl $\operatorname{PSM}(50: 50)$ binary mixture (see curve (a) in Fig. 4) is consistent with the POPC/PSM/Chol ternary phase diagram. ${ }^{9,40}$ Here, the passage from the $l_{\mathrm{d}}+s_{\mathrm{o}}$ two-phase region of the POPC/PSM (50:50) mixture to the $l_{\mathrm{o}}+l_{\mathrm{d}}$ two-phase region occurs at $\sim 4 \mathrm{~mol} \%$ Chol. Conversely, in the PSM/Chol system, the decrease in $C_{4}$ upon $\mathrm{Chol}$ addition starts to be felt at $\mathrm{mol} \% \mathrm{Chol}$ $>20$ and occurs over a broader $\mathrm{mol} \% \mathrm{Chol}$ range, as appears in curve (b) in Fig. 4.

\section{A modelistic interpretation}

In all the above measurements, the behavior of the capacity $C_{4}$ of the hydrocarbon tail region parallels that of the gel phase, by increasing when the gel phase increases and decreasing when it decreases. Moreover, the addition of $\mathrm{Chol}$ causes a decrease in $C_{4}$ that is faster in the presence of a $l_{\mathrm{d}}$ phase than in its absence. This behavior can be explained if we can assume that $C_{4}$ is approximately proportional to the total perimeter of the edge of the gel phase microdomains. As a matter of fact, the mismatch between these microdomains and the $l_{\mathrm{d}}$ phase is expected to provide a preferential pathway to the ions moving back and forth across the lipid monolayer, following the a.c. signal. This causes an increase in differential capacity and a decrease in resistance. In self-assembling a lipid monolayer of given composition on consecutive mercury drops, the reproducibility of the capacity is usually higher than that of the corresponding resistance. Therefore, attention will be mainly focused on the effect of lipid composition upon $C_{4}$.

The increase in the total perimeter of gel phase domains with an increase in the mole fraction of the high- $T_{\mathrm{m}}$ component depends not only on the number of microdomains but also on their average size at each mole fraction value. If several small microdomains were to merge into a larger one, the total perimeter of the microdomain edge would be reduced, as would the edge energy. Opposing this reduction in energy is the decreased entropy that would result from such a merger. In view of the competition between edge energy and entropy, at equilibrium the microdomain average size will attain a compromise value. Regarding the kinetics of microdomain formation and growth as a nucleation and growth process, entropy favors an increase in nucleation rate, while edge energy favors an increase in growth rate.

The balance between nucleation and growth rates in a binary lipid mixture can be treated using a model developed in Ref. 44 and based on the Avrami formalism. For simplicity, we will assume that the $s_{\mathrm{o}}$ phase is exclusively composed by the high- $T_{\mathrm{m}}$ component. Let us denote by $\theta$ the fraction of the unit surface area of the biomimetic membrane covered by the high- $T_{\mathrm{m}}$ lipid monomers and by $\theta_{0}$ its initial value, before the start of nucleation and growth; $\theta_{0}$ is, therefore, the whole mole fraction of the high- $T_{\mathrm{m}}$ lipid. According to the kinetic model of nucleation and growth in Ref. 44, the nucleation rate is given by:

$$
\mathrm{d} N / \mathrm{d} t=k_{\mathrm{N}} \theta^{n}
$$

Here $N$ is the number of nuclei per unit surface area, $k_{\mathrm{N}}$ is the nucleation rate constant and $n$ is the number of the high- $T_{\mathrm{m}}$ lipid monomers composing a critical nucleus. Assuming for simplicity that the growing supercritical clusters have a circular shape, their growth rate is given by:

$$
\frac{\mathrm{d}}{\mathrm{d} t}\left(\pi r^{2}\right)=2 \pi r \frac{\mathrm{d} r}{\mathrm{~d} t}=k_{\mathrm{R}} 2 \pi r \theta \rightarrow \frac{\mathrm{d} r}{\mathrm{~d} t}=k_{\mathrm{R}} \theta
$$

Here $r$ is the radius of the growing clusters and $k_{\mathrm{R}}$ is the rate constant of radial growth. The final average radius, $r_{\mathrm{f}}$, of the clusters is obtained from the equation:

$$
r_{\mathrm{f}}=k_{\mathrm{R}} \int_{\theta=\theta_{0}}^{\theta=0.001 \theta_{0}} \theta d t
$$

This integral is calculated numerically by determining $\theta$ as a function of time $t$ on the basis of the kinetic model, until it is reduced to one thousandth of its initial value, $\theta_{0}$. At this point, practically all the high- $T_{\mathrm{m}}$ lipid molecules participate in the formation of the gel phase microdomains.

The predictions of the kinetic model do not depend on the separate values of the rate constants $k_{\mathrm{N}}$ and $k_{\mathrm{R}}$, but on the $k_{\mathrm{N}}{ }^{1 / 2} k_{\mathrm{R}}$ product, which has the dimensions of $\left[t^{-3 / 2}\right]$ and does not contain the length. Therefore, the kinetic model predicts the 
functional dependence of the final average radius $r_{\mathrm{f}}$ of domains upon the mole fraction, $\theta_{0}$, of the high- $T_{\mathrm{m}}$ component, apart from a multiplicative factor. As expected, if $k_{\mathrm{N}}$ is much greater than $k_{\mathrm{R}}, r_{\mathrm{f}}$ decreases with an increase of $\theta_{0}$. Thus, for $n=5$ and $k_{\mathrm{N}}{ }^{1 / 2} k_{\mathrm{R}}=10 \mathrm{~s}^{-3 / 2}, r_{\mathrm{f}}$ decreases by a factor 0.48 when $\theta_{0}$ passes from 0.1 to 0.3 and by a factor 0.33 when $\theta_{0}$ passes from 0.1 to 0.5 . Conversely, when $k_{\mathrm{R}}$ is much greater than $k_{\mathrm{N}}, r_{\mathrm{f}}$ varies with $\theta_{0}$ only slightly. In practice, for $n=5$ and $k_{\mathrm{N}}{ }^{1 / 2} k_{\mathrm{R}}=100 \mathrm{~s}^{-3 / 2}$, $r_{\mathrm{f}}$ does not vary when $\theta_{0}$ passes from 0.1 to 0.5 . The number, $N_{\mathrm{d}}$, of microdomains per unit surface area at equilibrium is clearly given by;

$$
N_{d}=\theta_{0} / \pi r_{f}^{2}
$$

The total perimeter of the microdomain edges is equal to:

$$
2 \pi r_{f} N_{\mathrm{d}}=2 \theta_{0} / r_{f}
$$

Therefore, the total perimeter is expected to increase with $\theta_{0}$ more than linearly if $k_{\mathrm{N}}$ is much greater than $k_{\mathrm{R}}$, and linearly if $k_{\mathrm{N}}$ is much less than $k_{\mathrm{R}}$. The fact that $C_{4}$ shows no tendency to increase with $\theta_{0}$ more than linearly suggests that the growth rate prevails over the nucleation rate in determining the stationary size of the microdomains.

The Avrami formalism used in the above model excludes the overlapping of growing microdomains, but it does not account for their possible coalescence when they come into contact. As a rough approximation, we may assume that the probability that the total perimeter of the microdomain edges decreases by coalescence is inversely proportional to the surface area, $\left(1-\theta_{0}\right)$, free from the high- $T_{\mathrm{m}}$ component. With this assumption, the actual value of the total perimeter is obtained by multiplying its value in the absence of coalescence, as expressed by eqn (5), by $\left(1-\theta_{0}\right)$, yielding:

$$
2 \theta_{0}\left(1-\theta_{0}\right) / r_{f}
$$

If $r_{\mathrm{f}}$ does not depend upon $\theta_{0}$, this quantity attains a maximum for $\theta_{0}=1 / 2$. This may explain the slight decrease in $C_{4}$ with an increase in the PSM mol fraction that is observed at about $40 \mathrm{~mol} \% \mathrm{PSM}$ in the DOPC/PSM system (see Fig. 3).

The further increase in $C_{4}$ with an increase in the PSM mol fraction in Fig. 3 can also be explained by an increase in the perimeter of microdomains following the transition from the $s_{\mathrm{O}}+$ $l_{\mathrm{d}}$ to the $s_{\mathrm{o}}$ phase. In fact, the anisotropic gel phase can be regarded as consisting of microdomains with different orientations. Thus, 3D reconstructions of gel-phase DPPC GUVs exhibit a heterogeneous fluorescence intensity distribution, due to microdomain formation, as well as a deformation of their spherical shape. ${ }^{11}$ To distinguish these $s_{\mathrm{O}}$ microdomains, in contact with analogous microdomains with a different orientation, from the more familiar gel phase microdomains surrounded by a $l_{\mathrm{d}}$ phase, they will be referred to as "submicrodomains". As distinct from microdomains, submicrodomains do not have a domain-height mismatch. Fig. 3 shows that the formation of submicrodomains progressively richer in PSM is accompanied by an increase in their resistance $R_{4}$, thus exhibiting a behavior opposite to that accompanying the $l_{\mathrm{d}}$ to $l_{\mathrm{d}}+s_{\mathrm{o}}$ transition. Evidently, the tight packing of the $s_{\mathrm{o}}$ submicrodomains decreases ion permeation across them more than the length of their perimeters increases it. The attainment of a plateau of about $3.6 \mu \mathrm{F} \mathrm{cm} \mathrm{cm}^{-2}$ in the $C_{4} v s$. mol\% PSM plot between 70 and $90 \mathrm{~mol} \%$ PSM is in apparent contrast with the value of $\sim 6.5 \mu \mathrm{F} \mathrm{cm}^{-2}$ attained by pure PSM ( $c f$. curve (b) in Fig. 4 ). This behavior is ascribable to the small amount of the low- $T_{\mathrm{m}}$ phospholipid molecules of DOPC unavoidably present in the $s_{\mathrm{O}}$ microdomains; quite probably, these molecules are preferentially located at the junctions between adjacent $s_{\mathrm{o}}$ submicrodomains, thus decreasing the contribution of these junctions to the increase in capacity. The high capacity of pure PSM is attained only in close proximity of $100 \mathrm{~mol} \%$ PSM.

The effect of Chol in decreasing the capacity $C_{4}$ of the PSM/ Chol binary mixture starts to be felt only at $\mathrm{mol} \% \mathrm{Chol}>20$, as shown by curve (b) in Fig. 4. On the other hand, time-resolved FRET measurements on the PSM/Chol system point to a $s_{\mathrm{O}}$ to $s_{\mathrm{O}}+l_{\mathrm{o}}$ transition at $\sim 6.3 \mathrm{~mol} \% \mathrm{Chol}$ and to a subsequent $s_{\mathrm{o}}+l_{\mathrm{o}}$ to $l_{\mathrm{o}}$ transition at about $37 \mathrm{~mol} \% \mathrm{Chol}^{40}$ The latter transition was also reported at $30 \mathrm{~mol} \% \mathrm{Chol}$ by Halling et al. ${ }^{39}$ A similar behavior is reported for the thoroughly investigated DPPC/Chol system, ${ }^{41,45}$ where the $s_{\mathrm{O}}$ to $s_{\mathrm{O}}+l_{\mathrm{o}}$ transition occurs at $\sim 7 \mathrm{~mol} \%$ Chol and the $s_{\mathrm{o}}+l_{\mathrm{o}}$ to $l_{\mathrm{o}}$ transition at $\sim 30 \mathrm{~mol} \%$ Chol. To explain the absence of a detectable decrease in $C_{4}$ following the $s_{\mathrm{o}}$ to $s_{\mathrm{o}}+l_{\mathrm{o}}$ transition at about 6-7 $\mathrm{mol} \% \mathrm{Chol}$, we may hypothesize that the increase in capacity following the formation of $l_{\mathrm{o}}$ microdomains practically compensates for the decrease in capacity following the concomitant disappearance of a number of $s_{\mathrm{o}}$ submicrodomains.

It is noteworthy that the effectiveness of Chol in decreasing the capacity of the hydrocarbon tail region is greater when it is added to a $l_{\mathrm{d}}+s_{\mathrm{o}}$ two-phase region than when it is added to a $s_{\mathrm{o}}$ phase. Thus, $10 \mathrm{~mol} \% \mathrm{Chol}$ is sufficient to depress the capacity of the DOPC/PSM (50:50) binary mixture to its minimum value, as shown by curve (a) in Fig. 4. Conversely, more that $50 \mathrm{~mol} \%$ Chol is required to depress the capacity of the PSM/Chol mixture to the same minimum value (see curve (b) in Fig. 4). This behavior can again be justified by the small amount of DOPC molecules present in the $s_{\mathrm{o}}$ phase; these are probably intercalated along the boundaries of the $s_{\mathrm{O}}$ submicrodomains, thus abating the increase in capacity due to these boundaries. In this case, the main effect of Chol addition is that of eliminating the mismatch at the boundary between the anisotropic $s_{\mathrm{O}}$ microdomains and the surrounding $l_{\mathrm{d}}$ phase, by converting these microdomains into lipid rafts.

\section{Conclusion}

In conclusion, $\mathrm{Hg}$-supported monolayers and bilayers of lipid mixtures form spontaneously microdomains analogous to those formed in free standing bilayer lipid membranes. The differential capacity of the hydrocarbon tail region of lipid mixtures is sensitive to phase transitions, thus permitting these transitions in Hg-supported lipid monolayers to be monitored by EIS. In addition, the conclusions as to the changes in capacitance and resistance of lipid monolayers in passing from the liquid-disordered to the liquid-ordered phase and from this to the gel phase can be extended to the distal leaflet of biological membranes; this provides additional information on the physico-chemical properties of these coexisting phases. Imaging of lipid phases in the 
distal monolayer of $\mathrm{Hg}$-supported lipid bilayers can be obtained by fluorescence lifetime spectroscopy.

\section{Acknowledgements}

The financial support by Ente Cassa di Risparmio di Firenze and by the Italian Ministero dell'Istruzione, dell'Università e della Ricerca (MIUR) through PRIN 20079Y9578 are gratefully acknowledged.

\section{References}

1 D. A. Brown and E. London, J. Membr. Biol., 1998, 164, 103-114.

2 D. A. Brown and E. London, J. Biol. Chem., 2000, 275, 17221-17224.

3 T. Parasassi, A. M. Giusti, M. Raimondi and E. Gatton, Biophys. J., 1995, 68, 1895-1902.

4 L. A. Bagatolli, Biochim. Biophys. Acta, Biomembr., 2006, 1758, 1541-1556.

5 K. Simons and E. Ikonen, Nature, 1997, 387, 569-72.

6 T. Parasassi, E. Gratton, W. M. Yu, P. Wilson and M. Levi, Biophys. J., 1997, 72, 2413-2429.

7 L. A. Bagatolli and E. Gratton, J. Fluoresc., 2001, 11, 141-160.

8 R. F. M. de Almeida, J. W. Borst, A. Fedorov, M. Prieto and A. J. W. G. Visser, Biophys. J., 2007, 93, 539-553.

9 R. F. M. de Almeida, L. M. S. Loura and M. Prieto, Chem. Phys. Lipids, 2009, 157, 61-77.

10 S. N. Ahamed, D. A. Brown and E. London, Biochemistry, 1997, 36, 10944-10953.

11 F. S. Ariola, D. J. Mudaliar, R. P. Walvick and A. A. Heikal, Phys. Chem. Chem. Phys., 2006, 8, 4517-4529.

12 N. Kucerca, J. Pencer, M.-P. Nieh and J. Katsaras, Eur. Phys. J. E, 2007, 23, 247-254.

13 A. V. Samsonov, I. Mihalyov and F. S. Cohen, Biophys. J., 2001, 81, 1486-1500.

14 C. Dietrich, L. A. Bagatolli, Z. N. Volovyk, N. L. Thompson, M. Levi, K. Jacobson and E. Gratton, Biophys. J., 2001, 80, 14171428.

15 K. Gaus, E. Gratton, E. P. W. Kable, A. S. Jones, I. Gelissen, L. Kritharides and W. Jessup, Proc. Natl. Acad. Sci. U. S. A., 2003, 100, 15554-15559.

16 J. C. Lawrence, D. E. Saslowsky, J. M. Edwardson and R. M. Henderson, Biophys. J., 2003, 84, 1827-1832.

17 C. Yuan and L. J. Johnston, Biophys. J., 2000, 79, 2768-2781.

18 C. Yuan, J. Furlong, P. Burgos and L. J. Johnston, Biophys. J., 2002, 82, 2526-2535.

19 A. J. García-Sáez, S. Chiantia and P. Schwille, J. Biol. Chem., 2007, 282, 33537-33544.
20 M. Naumowicz and Z. A. Figaszewski, Biophys. J., 2005, 89, 3174-3182.

21 M. Naumowicz, A. D. Petelska and Z. A. Figaszewski, Electrochim. Acta, 2005, 50, 2155-2161.

22 M. Naumowicz and Z. A. Figaszewski, J. Membr. Biol., 2005, 205, 29-36.

23 A. Nelson and A. Benton, J. Electroanal. Chem., 1986, 202, 253-270.

24 M. R. Moncelli, L. Becucci and R. Guidelli, Biophys. J., 1994, 66, 1969-1980.

25 M. R. Moncelli, L. Becucci, A. Nelson and R. Guidelli, Biophys. J., 1996, 70, 2716-2726.

26 L. Becucci, M. R. Moncelli, R. Naumann and R. Guidelli, J. Am. Chem. Soc., 2005, 127, 13316-13323.

27 L. Becucci, M. R. Moncelli and R. Guidelli, Langmuir, 2006, 22, 1341-1346.

28 L. Becucci, M. V. Carbone, T. Biagiotti, M. D'Amico, M. Olivotto and R. Guidelli, J. Phys. Chem. B, 2008, 112, 1315-1319.

29 L. Becucci, R. Guidelli, C. B. Karim, D. D. Thomas and G. Veglia, Biophys. J., 2007, 93, 2678-2687.

30 L. Becucci, A. Cembran, C. B. Karim, D. D. Thomas, R. Guidelli, J. Gao and G. Veglia, Biophys. J., 2009, 96, L60-L62.

31 A. C. Pineda and D. Ronis, J. Chem. Phys., 1985, 83, 5330-5337.

32 M. R. Moncelli and L. Becucci, J. Electroanal. Chem., 1997, 433, 91-96.

33 L. Becucci, M. D’Amico, S. Daniele, M. Olivotto, A. Pozzi and R. Guidelli, Bioelectrochemistry, 2010, 78, 176-180.

34 M. Montal and P. Mueller, Proc. Natl. Acad. Sci. U. S. A., 1972, 69, 3561-3566.

35 A. Nelson and N. Auffret, J. Electroanal. Chem., 1988, 244, 99-113.

36 R. Guidelli in Encyclopedia of Electrochemistry, Vol. 10, Modified Electrodes, Ed. M. Fujihira, I. Rubinstein and J. F. Rusling, WileyVCH, New York, 2007, pp.105-157.

37 D. Bizzotto, Y. Yang, J. Shepherd, R. Stoodley, J. Agak, V. Stauffer, M. Lathuillière, A. S. Akhtar and E. Chung, J. Electroanal. Chem., 2004, 574, 167-184.

38 L. Becucci, M. Innocenti, E. Salvietti, A. Rindi, I. Pasquini, M. Vassalli, M. L. Foresti and R. Guidelli, Electrochim. Acta, 2008, 53, 6372-6379.

39 K. K. Halling, B. Ramstedt, J. H. Nyström, J. P. Slotte and T. K. M. Nyholm, Biophys. J., 2008, 95, 3861-3871.

40 R. F. M. de Almeida, L. M. S. Loura, A. Fedorov and M. Prieto, J. Mol. Biol., 2005, 346, 1109-1120.

41 J. R. Silvius, D. del Giudice and M. Lafleur, Biochemistry, 1996, 35, 15198-15208.

42 S. L. Veatch, K. Gawrisch and S. L. Keller, Biophys. J., 2006, 90, 4428-4436.

43 O. Bakht, P. Pathak and E. London, Biophys. J., 2007, 93, 4307-4318.

44 L. Becucci, M. R. Moncelli and R. Guidelli, J. Am. Chem. Soc., 2003, 125, 3784-3792.

45 M. B. Sankaram and T. E. Thompson, Proc. Natl. Acad. Sci. U. S. A., 1991, 88, 8686-8990. 